\title{
Palliative Radiation Therapy of Symptomatic Recurrent Bladder Cancer
}

\author{
Sun K. Yi', Mark Yoder², Ken Zaner², MD, PhD, and Ariel E. Hirsch', MD
}

From: Departments of ${ }^{1}$ Radiation Oncology and ${ }^{2}$ Medical Oncology Boston University Medical Center, Boston, MA

$\mathrm{Yi}^{1}$ is a fourth year medical student, Department of Radiation Oncology, Boston University School of Medicine, Boston, MA

Yoder $^{2}$ is a Nurse Practioner, Department of Hematology/ Oncology, Boston University Medical Center, Boston, MA

Dr. Zaner ${ }^{2}$ is Associate Professor of Medicine, Department of Hematology/ Oncology, Boston University Medical Center, Boston, MA

Dr. Hirsch ${ }^{1}$ is Assistant Professor, Director of Education, Department

of Radiation Oncology, Boston

University Medical Center, Boston,

MA; and Assistant Radiation

Oncologist, Massachusetts General

Hospital, Harvard Radiation

Oncology Program, Boston, MA

Address Correspondence:

Ariel E. Hirsch, MD

Department of Radiation Oncology

Boston University Medical Center

830 Harrison Avenue

Moakley Building-Lower Leve

Boston, MA 02118

E-mail: ariel.hirsch@bmc.org Funding: None.

Conflict of Interest: None.

Manuscript received: 11/28/2006

Revisions received: $1 / 3 / 2007$

Accepted for Publication: 1/22/2007

Free Full manuscript: www.painphysicianjournal.com
Background: Palliative radiation therapy (RT) is an established tool in the management of symptoms caused by malignancies. RT is effective at palliating both locally advanced and metastatic cancer, including related symptoms of pain, bleeding, or obstruction. Most data on palliative RT is in regard to its use in the treatment of painful bone metastases. There are also data that support RT palliation for locally advanced or recurrent rectal, prostate, and gynecological cancers. With regard to bladder cancer there is some evidence of the benefit of palliative RT for the control of urinary symptoms and hematuria; however, there is little evidence for the use of palliative RT for pain associated with locally recurrent bladder cancer. We report a case of locally advanced recurrent bladder cancer which was refractory to medical pain management, and was found to be highly responsive to palliative RT.

Case Report: An 80-year-old woman with recurrent bladder cancer and intractable pelvic pain refractory to oral and transdermal pain medications, received palliative pelvic RT to a dose of 50 Gy (5000 cGy) in 25 fractions with complete resolution of pain. The patient was originally found to have dysuria, frequency, and hematuria, secondary to an invasive high grade transitional cell carcinoma of the bladder with an adenocarcinoma component, AJCC pT2b N1 MO Stage IV, for which she underwent a radical cystectomy, total abdominal hysterectomy, bilateral salpingo-oophorectomy, partial vaginectomy, and ileal conduit reconstruction. After undergoing 4 cycles of adjuvant chemotherapy, the patient did well for 5 months with no evidence of symptomatic, clinical, or radiographic recurrence of disease. Repeat staging CT of the abdomen and pelvis confirmed tumor recurrence in the left pelvis. The patient was treated with another course of chemotherapy and pain was managed with relatively low doses of opioid medication (25 mcg transdermal fentanyl patch, and oxycodone $5 \mathrm{mg}$ bid). However at the fourth month, there was rapid escalation of severe pain with the patient becoming bed bound due to pain with an associated decrease in ambulation and anorexia. Ultimately a pain medication regimen of $200 \mathrm{mcg}$ transdermal fentanyl patch q2 days, oxycontin $20 \mathrm{mg}$ bid, oxycodone $5-10 \mathrm{mg}$ q 4 hours, ibuprofen $400 \mathrm{mg} \mathrm{q} 8$ hours, and gabapentin $600 \mathrm{mg}$ TID was not effective in controlling pain. The patient was then referred to Radiation Oncology 6 months after the pain initially began for evaluation. She received a total of 5000cGy over 25 fractions to a small pelvis field over 5 weeks and reported complete pain resolution. She was able to decrease pain medications, increase overall activity, and gain significant improvement in sleep quality and appetite even early on in the course of her radiation therapy.

Conclusions: Palliative radiation therapy has been well studied in the setting of bone metastases and treatment of hematuria for locally advanced bladder cancer. There is little data that we are aware of on the use of RT for pain control with patients that have recurrent, locally advanced bladder cancer. We have presented a case in which an excellent outcome in pain control was seen for a patient with medically unmanageable pain. RT is an excellent option for pain management in recurrent bladder cancer and should be offered to patients whose pain is not otherwise optimally controlled. Palliative RT is an important component in the multimodality approach to cancer pain management and optimization of quality of life.

Key words: palliation, bladder cancer, radiation therapy

Pain Physician 2007; 10:285-290 
A n 80-year-old woman was initially diagnosed with a transitional cell carcinoma (TCC) with an adenocarcinoma component of the bladder in February 2005 upon evaluation by cystoscopy and transurethral resection, after complaining of dysuria, frequency, and hematuria. In March 2005, the patient underwent radical cystectomy, total abdominal hysterectomy, bilateral salpingo-oophorectomy, partial vaginectomy, and ileal conduit reconstruction for a $3 \times 2.5 \times 1.2 \mathrm{~cm}$, invasive high grade TCC with a major glandular differentiated (adenocarcinoma) component, found extending through the muscular wall ( $2 \mathrm{~mm}$ from serosal surface). One out of 8 dissected lymph nodes were positive for metastatic TCC from the right external iliac and obturator region (AJCC Pathologic Stage IV pT2b N1 M0).

The patient was seen by medical oncology in April 2005 following her surgery and received adjuvant cisplatinum and gemcitabine chemotherapy for 4 cycles, which the patient tolerated well. Upon completion of chemotherapy, a CT of the abdomen and pelvis was taken in August 2005, and showed no evidence for recurrence. The patient reported doing well until December 2005 when she reported left lower quadrant pain.

The patient described the pain as "pressure-like" in quality, radiating to the low back, and was made worse with standing and relieved with sitting or lying. The patient reported gradual worsening of the pain over time, which was found to be exacerbated by eating and associated with constipation. While hospitalized for increasing pain, the patient underwent a repeat CT scan of the abdomen and pelvis, that showed soft tissue masses located within the left pelvis that were suggestive of tumor recurrence.

The patient subsequently received Taxotere $\times 3$ cycles in the months of February and March of 2006. The patient was initially placed on morphine elixir for pain management and after 1 dose experienced severe epigastric pain, vomiting, and chest pain resulting in hospitalization. A complete cardiac workup was negative and the symptoms were thought to be due to the side effects from the morphine elixir. The patient was discharged from the hospital on morphine sustained release tablets $15 \mathrm{mg}$ PO BID and oxycodone $5 \mathrm{mg}$ PO BID PRN for breakthrough pain.

The patient was seen in the medical oncology clinic for pain reevaluation 1 week following her discharge. At that time, she reported restlessness, unsteadiness on her feet, and picking at things in the air. This was thought to be related to the morphine and the sustained release morphine was discontinued. She was then started on $25 \mathrm{mcg}$ fentanyl transdermal patch changed q 48 hours. The patient's pain was closely monitored over the subsequent few months with gradual titration of fentanyl transdermal patch to $75 \mathrm{mcg}$ q2d and oxycodone was increased to $10 \mathrm{mg}$ PO q4-6h PRN for breakthrough pain.

$A$ repeat $C T$ of the abdomen and pelvis in May 2006 showed interval worsening of her left-sided pelvic soft tissue mass without evidence of bony abnormality (Fig. 1). She was now starting to experience severe pain without relief even with the increase in Fentanyl dose to $150 \mathrm{mcg} q 2 \mathrm{~d}$. At this point the patient reported that the pain was interfering with her sleep and appetite. Additionally, at that time she had become virtually bed bound, because it was too painful to walk leading to a subsequent decrease in physical strength, and she required weekly visits to the oncology clinic for hydration.

The patient was then referred to the radiation oncology department in June 2006, at which point she was on duragesic fentanyl patch $200 \mathrm{mcg}$ q2d, gabapentin $300 \mathrm{mg}$ PO QID, ibuprofen $400 \mathrm{mg}$ q6h, and oxycontin 20mg PO BID for severe (7/10 on the numeric pain scale) pain with no relief at all.

Radiation therapy commenced on June 1, 2006, for 25 fractions to a total dose of $5000 \mathrm{cGy}$ to a small pelvis field (Fig. 2). After the initial 2000 cGy out of total 5000 cGy the patient reported improvement in pain. The patient was able to decrease her fentanyl patch to $150 \mathrm{mcg}$, without need for oxycontin. With subsequent radiation treatment the patient continued to report increasing pain relief, increased activity, improvement in sleep, and improved appetite. Halfway through the course of radiation, the patient was able to decrease her fentanyl patch to 125 mcg with no need for breakthrough pain medication. At that point in time, the patient denied any pelvic pain (0/10). Upon completion of the 25 fractions of radiation, the patient continued to report no pain (0/10) and was able to decrease her fentanyl patch to $75 \mathrm{mcg}$, with no need for breakthrough pain medication. 


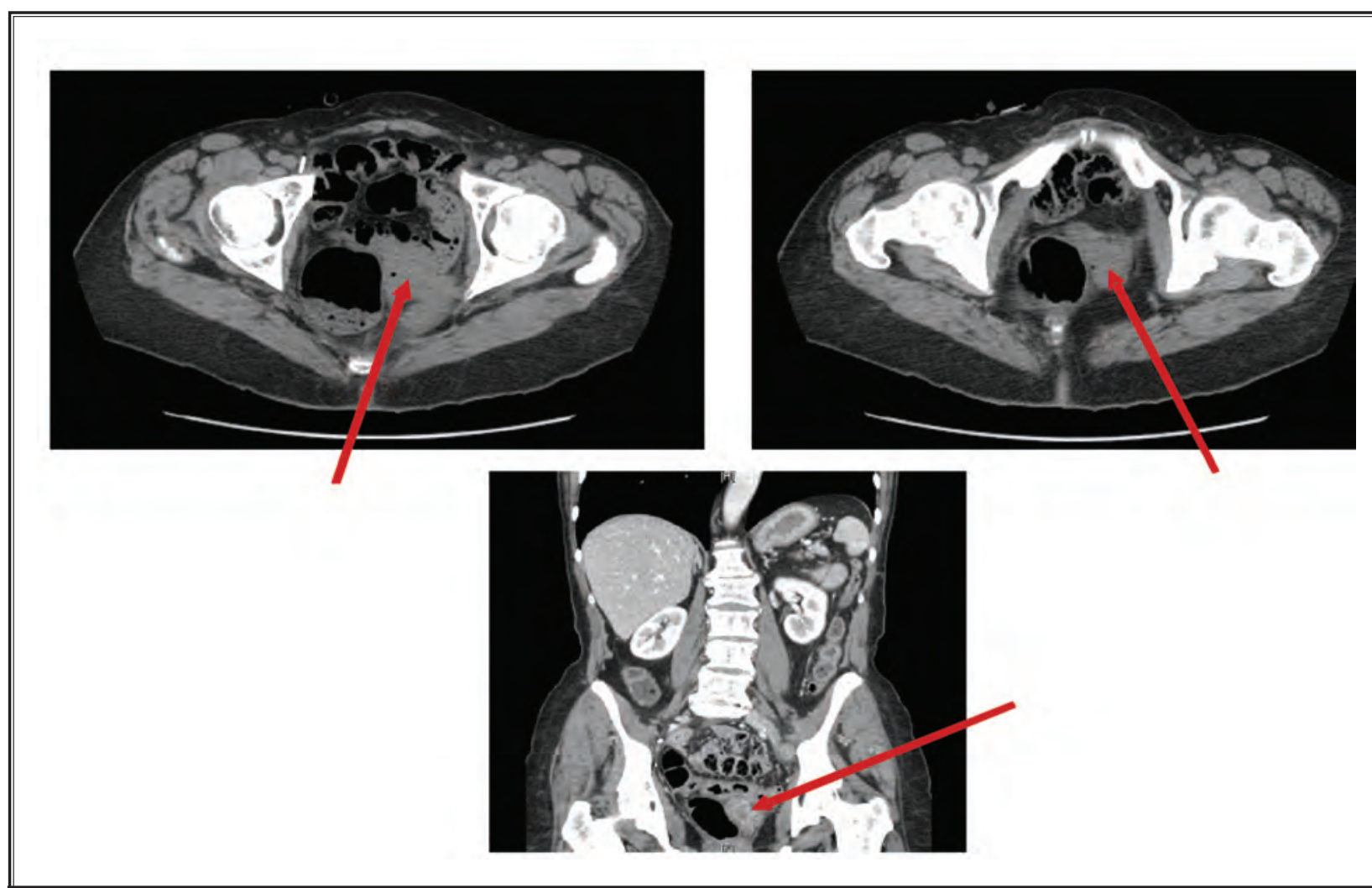

Fig. 1. May 2006 CT scan of abdomen and pelvis showing large soft tissue densities in the left pelvis causing mass effect of the rectum (tumor indicated with red arrows).

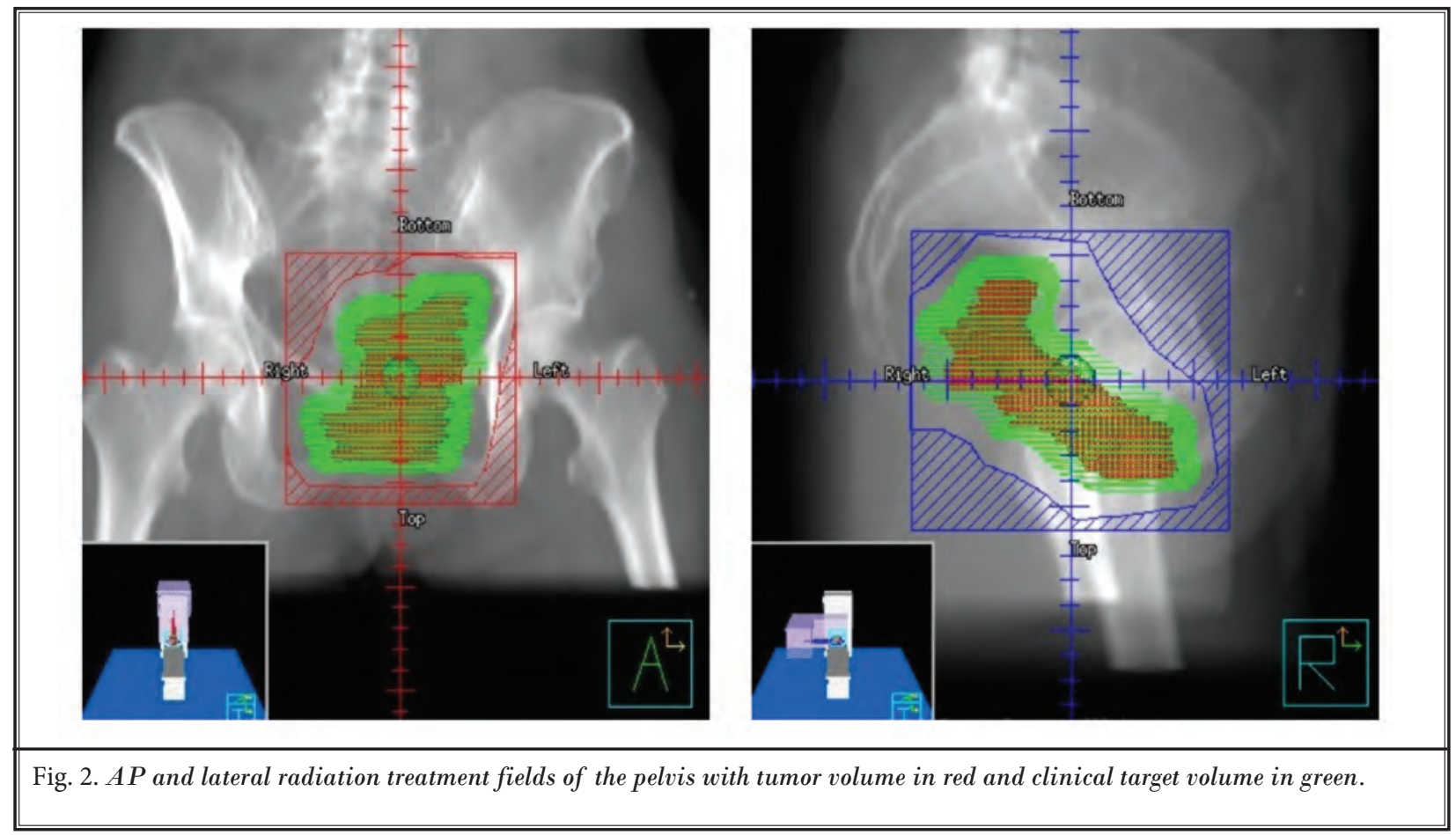




\section{Discussion}

Pain is one of the most feared symptoms encountered by patients who suffer from cancer (1). The Radiation Therapy Oncology Group (RTOG) has reported that many physicians, as high as $83 \%$ of RTOG-affiliated physicians according to 1 recently taken survey, believe that pain is undertreated in cancer patients for a variety of reasons including inadequate pain assessment, underreporting of pain by the patient, fear of opioid dependence, as well as other socio-legal issues (2). The American Pain Society (APS) has provided well-documented guidelines for pain management of the cancer patient. Much of the guidelines has been geared toward the pharmacological treatment of pain associated with cancer including the use of NSAIDS, opioids, and anticonvulsant medications. Palliative RT is only briefly mentioned in the APS guideline for management of cancer pain (3). Recent APS recommendations have stressed the importance of employing a multimodality treatment approach to the treatment of cancer pain (4).

With respect to palliative RT in the current literature, there are well-established data that describe the benefit of palliative radiation therapy for pain, bleeding, or obstruction associated with recurrent, locally advanced, or metastatic cancer $(2,5,6)$. RT for the palliative treatment and management of painful bone metastases has been well studied and has been shown to be extremely effective reducing pain in up to $90 \%$ of patients (7-11). Currently external beam radiation therapy is the standard of care for treating patients suffering from bone metastases, and recent studies looking at radiopharmaceuticals, such as strontium89 , have also shown a benefit in pain management (12).

With regard to the palliative management of pelvic malignancies, there are data that suggest a benefit of palliative RT treatment for locally advanced tumors, particularly rectal and gynecological cancers. Studies that have evaluated the benefit of palliative RT for rectal cancer have shown statistically significant improvement in pain control for patients suffering from locally advanced disease (13-16). Improvement in pain control has also been found with palliative RT in patients with locally advanced gynecological malignancies including cervical, endometrial, and ovarian cancers (17-20). Furthermore, there are data that have shown palliative RT to be effective in the pain relief of patients suffering from advanced, hormone-refractory prostate cancer $(21,22)$.
In terms of palliative RT for the treatment of symptoms associated with recurrent and locally advanced bladder cancer, most of the literature describes improvement in malignancy-associated hematuria (23-25). There have been few studies that have specifically addressed the role of RT for pain relief in patients who suffer from recurrent bladder cancer. In 1 study performed by Srinivasan et al (26), a comparison of 2 radiotherapy regimens for the treatment of advanced bladder cancer, in which one group received 45 Gy over 12 fractions compared to $17 \mathrm{~Gy}$ in 2 fractions, showed pain improvement in both groups, 37\% and $73 \%$, respectively.

In our case, the patient's gradual worsening of pain was initially managed with standard medical management using opioids. Once the patient began to experience a marked escalation of pain, the pain medications were titrated accordingly by the guidelines provided by the APS and eventually included a regimen of opioids, NSAIDs, and an anticonvulsant. Her pain was found to be refractory to the increase in doses that were given to her at regular follow-up visits. Her pain control with medical management was particularly challenging as she had a number of opioid-related side-effects, including severe epigastric pain with only $5 \mathrm{mg}$ oral morphine elixir requiring hospital admission and full cardiac workup, visual hallucinations with extended release morphine tablets, and severe oxycodone-related drowsiness.

When the patient was evaluated in the radiation oncology department 6 months following the initiation of her pain, the patient reported severe, 7/10, "pressure-like" pain, which radiated to her lower back and was made worse with standing, but relieved with sitting or lying down. A CT scan of the abdomen and pelvis showed recurrent bladder cancer (Fig. 1) that was increasing in size and causing a mass effect to surrounding pelvic structures. In addition to gradually worsening pain, our patient suffered from worsening fatigue and inactivity, increased sleep disturbance, and decreased appetite secondary to her pain. Over the first 4 months immediately preceding referral to radiation therapy, the patient's pain symptoms were medically managed with variable results. However, over the 1 month just prior to referral to radiation, the symptoms rapidly escalated with severe pain leading to bed bound status and anorexia. She required weekly IV hydration to maintain nutritional status. The fentanyl dose had increased from $25 \mathrm{mcg}$ to 200 $\mathrm{mcg}$ in this time period with little relief. 
The patient received palliative RT to the left pelvis to a total of 5000 cGy in 25 fractions over 5 weeks to a small pelvis field (Fig. 2). After only the first 2 weeks of palliative RT the patient noted complete resolution of her pain, $0 / 10$ in severity on the numeric pain scale. She reported minimal side effects directly attributable to RT, only reporting mild loose stools, which the patient actually welcomed since the constipation induced by increasing opioid intake was thought to be contributory to her postprandial abdominal pain and anorexia.

The mechanism of action for palliative RT and decreased pain symptoms is not completely understood at this time. It is believed that pain relief from external beam radiation may be secondary to the tumoricidal effect of RT on cells from advanced malignancy. It is thought that deceleration of tumor growth may allow for decreased surrounding edema, and therefore, decreased pressure on pain nerve fibers, thus allowing for decreased neuropathic or nociceptive pain stimulus. Others believe that RT-associated pain reduction occurs through alterations in the pain signaling pathways, and current animal model research is actively underway to elucidate the mechanisms for which cancer pain occurs and is altered by modalities such as RT $(27,28)$. While the mechanism of action remains unknown, RT has been shown to reduce cancer related pain, in addition to increasing the quality of life of patients who suffer from advanced cancer.

One goal of adequate pain management in patients with advanced cancer is to increase the Health-Related Quality of Life (HRQOL) for terminally ill patients (29). Palliative treatment for cancer patients is no longer regarded simply as the supportive means by which to reduce undesirable symptoms, but is better recognized as the World Health Organization defines it, "the active, total care of a person whose condition is not responsive to curative treatment" (30). That being said, it is important that we effectively treat pain and other unwanted symptoms associated with locally advanced cancers to improve the overall HRQOL of the patient.
Quality of life and its relationship to prognosis and/or survival rates has been studied in a variety of cancers and the data suggest that there may be a positive correlation between quality of life and prognosis/survival (31-34). When our patient was seen in the radiation oncology department, her HRQOL was poor due to her intractable pelvic pain, increasing fatigue and anorexia, and decreased ability to sleep and perform daily activities. She was confined to a wheelchair for mobility, and even getting up on the exam table was extremely challenging for her, as the pelvic pain was so severe. As our patient underwent palliative RT to her left pelvis, she reported complete pain resolution as well as overall increase in her HRQOL. We believe that early palliative RT included in the management of locally advanced recurrent bladder cancer is an important component of pain management and optimization of quality of life. More studies are needed to specifically evaluate the effectiveness of palliative RT for pain management and optimization of HRQOL in patients who suffer from recurrent, locally advanced bladder cancer.

\section{Conclusion}

Palliative radiation therapy has been well studied and there are well-established data for the benefit of palliative RT in the setting of bone metastases and locally advanced malignancy. Although there are studies that support the use of palliative RT for the treatment of hematuria for locally advanced bladder cancer, there are little data that we are aware of that have examined the use of RT for pain control with patients who have recurrent, locally advanced bladder cancer. We have presented a case in which an excellent outcome in pain control and increased HRQOL was seen for a patient with medically unmanageable pain. RT is an excellent option for pain management in recurrent bladder cancer and should be offered to patients whose pain is not otherwise optimally controlled, particularly in elderly patients who are sensitive to opioid therapy. 


\section{References}

1. Foley KM, Gelband H. Improving palliative care for cancer: Summary and recommendations. Washington, DC: National Academies Press 2001.

2. Konski A, Feigenberg S, Chow E. Palliative radiation therapy. Seminars in Oncology. 2005; 32:156-64.

3. Miaskowski C, Cleary J, Burney R, Coyne P, Finley R, Foster R, Grossman S, Janjan N, Ray J, Syrjala K, Weisman S, Zahrbock C. Guideline for the management of cancer pain in adults and children, American Pain Society Clinical Practice Guidelines Series, No. 3. Glenview, IL: 2005.

4. Gordon DB, Dahl JL, Miaskowski C, McCarberg B, Todd KH, Paice JA, Lipman AG, Bookbinder M, Sanders SH, Turk DC, Carr DB. American pain society recommendations for improving the quality of acute and cancer pain management: American Pain Society Quality of Care Task Force. Archives of Internal Medicine 2005; 165:1574-1580.

5. Hoegler D. Radiotherapy for palliation of symptoms in incurable cancer. Current Problems in Cancer 1997; 21:129183.

6. Dolinsky C, Metz JM. Palliative radiation therapy in oncology. Anesthesiology Clinics 2006; 24:113-128, viii-ix.

7. Cleeland CS, Janjan NA, Scott CB. Seiferheld WF. Curran WJ. Cancer pain management by radiotherapists: a survey of radiation therapy oncology group physicians. International Journal of Radiation Oncology, Biology, Physics 2000; 47:203-208.

8. Tong D, Gillick L, Hendrickson FR. The palliation of symptomatic osseous metastases: final results of the Study by the Radiation Therapy Oncology Group. Cancer 1982; 50:893-899.

9. Falkmer U, Jarhult J, Wersall P, CavallinStahl E. A systematic overview of radiation therapy effects in skeletal metastases. Acta Oncologica 2003; 42:620633.

10. McQuay HJ, Collins SL, Carroll D. Moore RA. Radiotherapy for the palliation of painful bone metastases. Cochrane $\mathrm{Da}$ tabase of Systematic Reviews 2000; (2):CDoo1793.

11. Ratanatharathorn V. Powers WE. Moss WT. Perez CA. Bone metastasis: review and critical analysis of random allocation trials of local field treatment. International Journal of Radiation Oncology, Biology, Physics 1999; 44:1-18.

12. Bauman G, Charette M, Reid R, Sathya
J. Radiopharmaceuticals for the palliation of painful bone metastasis-a systematic review. Radiotherapy \& Oncology 2005; 75:258-270.

13. Allum WH, Mack P, Priestman TJ. Fielding JW. Radiotherapy for pain relief in locally recurrent colorectal cancer. Annals of the Royal College of Surgeons of England 1987; 69:220-221.

14. Wong R, Thomas G, Cummings B, Froud P, Shelley W, Withers RH, Williams IJ. The role of radiotherapy in the management of pelvic recurrence of rectal cancer. Canadian Journal of Oncology 1996; 6 Suppl 1:39-47.

15. Wong R, Thomas G, Cummings B, Froud $P$, Shelley W, Withers R, Williams J. In search of a dose-response relationship with radiotherapy in the management of recurrent rectal carcinoma in the pelvis: a systematic review. International Journal of Radiation Oncology, Biology, Physics 1998; 40(2):437-46,

16. Ito Y, Ohtsu A, Ishikura S, Boku N, Nihei $\mathrm{K}$, Ogino T, Ikeda H. Efficacy of chemoradiotherapy on pain relief in patients with intrapelvic recurrence of rectal cancer. Japanese Journal of Clinical Oncology 2003; 33:180-185.

17. Smith SC, Koh WJ. Palliative radiation therapy for gynaecological malignancies. Best Practice \& Research in Clinical Obstetrics \& Gynaecology 2001; 15:265-278.

18. Onsrud M, Hagen B, Strickert T. 10Gy single-fraction pelvic irradiation for palliation and life prolongation in patients with cancer of the cervix and corpus uteri. Gynecologic Oncology 2001; 82:167-171.

19. Adelson MD, Wharton JT, Delclos L, Copeland L, Gershenson D. Palliative radiotherapy for ovarian cancer. International Journal of Radiation Oncology, Biology, Physics 1987; 13:17-21.

20. Tinger A, Waldron T, Peluso N, Katin MJ, Dosoretz DE, Blitzer PH, Rubenstein JH, Garton GR, Nakfoor BA, Patrice SJ, Chuang L, Orr JW Jr. Effective palliative radiation therapy in advanced and recurrent ovarian carcinoma. International Journal of Radiation Oncology, Biology, Physics 2001; 51:1256-1263.

21. C atton CN, Gospodarowicz MK. Palliative radiotherapy in prostate cancer. Seminars in Urologic Oncology;1997; 15:6572.

22. Kynaston HG, Keen CW, Matthews PN. Radiotherapy for palliation of locally advanced prostatic carcinoma. British
Journal of Urology 1990; 66:515-517.

23. Fossa SD. Pelvic palliation radiotherapy of advanced bladder cancer. International Journal of Radiation Oncology, Biology, Physics 1991; 20:1379.

24. Petrovich Z, Jozsef G, Brady LW. Radiotherapy for carcinoma of the bladder: a review. American Journal of Clinical Oncology 2001; 24:1-9.

25. McLaren DB, Morrey D, Mason MD. Hypofractionated radiotherapy for muscle invasive bladder cancer in the elderly. Radiotherapy \& Oncology 1997; 43:171174.

26. Srinivasan $\mathrm{V}$, Brown $\mathrm{CH}$, Turner AG. A comparison of two radiotherapy regimens for the treatment of symptoms from advanced bladder cancer. Clinical Oncology (Royal College of Radiologists) 1994; 6:11-13.

27. Mantyh PW, Clohisy DR, Koltzenburg M,Hunt SP. Molecular mechanisms of cancer pain. Nature Reviews- Cancer 2002; 2:201-209.

28. Seong J, Park HC, Kim J, Kim UJ, Lee BW. Radiation-induced alteration of pain-related signals in an animal model with bone invasion from cancer. Annals of the New York Academy of Sciences 2004; 1030:179-186.

29. www.cdc.gov/hrqol/index.htm

30. World Health Organization. Cancer pain relief and palliative care. Report of a WHO Expert Committee. World Health Organization Technical Report Series 1990; 804:1-75.

31. Coates A, Porzsolt F, Osoba D. Quality of life in oncology practice: prognostic value of EORTC QLQ-C30 scores in patients with advanced malignancy. European Journal of Cancer 1997; 33:10251030.

32. Vigano A, Donaldson N, Higginson IJ, Bruera E, Mahmud S, Suarez-Almazor M. Quality of life and survival prediction in terminal cancer patients: a multicenter study. Cancer 2004; 101:10901098.

33. Lis CG, Gupta D, Granick J, Grutsch JF. Can patient satisfaction with quality of life predict survival in advanced colorectal cancer? Support Care Cancer 2006; 14:1104-1110

34. Kim A. Fall P. Wang D. Palliative care: optimizing quality of life. Journal of the American Osteopathic Association 2005; 105 (11 Suppl 5):S9-14. 